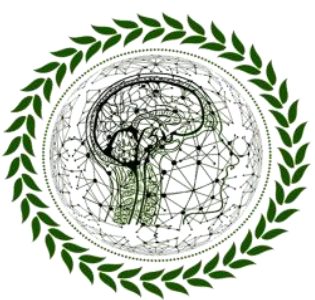

PhI Scientific Review

ISSN 2676 - 0444

Submetido em: 04/12/2021 | Aceito em: 07/12/2021 | Publicado em: 16/12/2021 | Artigo

\title{
ATIVIDADE FÍSICA, HÁBITOS SAÚDAVEIS E OBESIDADE EM CRIANÇAS E ADOLESCENTES: CONSIDERAÇÕES, RECOMENDAÇÕES E INTERVENÇÕES.
}

\author{
Gustavo Baroni Araujo ${ }^{1}$ \\ Célio Pereira de Sousa Júnior ${ }^{2}$ \\ Yasmin Emanuelly Leal Araújo ${ }^{3}$ \\ Thyago de Oliveira Afonso ${ }^{4}$ \\ João Felipe Tinto Silva ${ }^{5}$ \\ Renato Amaral Pereira ${ }^{6}$
}

\begin{abstract}
Resumo: A obesidade é uma doença cada vez mais comum, cuja prevalência já atinge proporções epidêmicas. $\mathrm{O}$ estilo de vida ativo e a adoção de hábitos saudáveis mostraram-se determinantes no atual quadro da obesidade infantil. Nesse sentido, considera-se a obesidade como uma doença multifatorial, devendo ser tratada de forma multiprofissional. O objetivo deste trabalho é discutir aspectos gerais, recomendações e intervenções no que diz respeito a atividade física e hábitos saudáveis em crianças e adolescentes com obesidade. Trata-se de uma revisão de literatura, realizada nas bases de dados Scientific Electronic Library Online (SciELO) e Centro Latino-Americano e do Caribe de Informação em Ciências da Saúde (Bireme). Os artigos foram selecionados a partir das seguintes palavras-chave em língua portuguesa: obesidade infantil; recomendações; cuidados. Os critérios de inclusão foram estudos com crianças e adolescentes que apresentassem um quadro de sobrepeso ou obesidade que apresentassem relação com a prática do exercício físico, hábitos alimentares, intervenções multiprofissionais. Foram selecionados apenas artigos publicados entre os anos de 2012 a 2020 e apenas da língua portuguesa e língua inglesa. No total, 12 artigos compuseram esta revisão. Constatou-se que a obesidade é um processo multifatorial que envolve questões ambientais, culturais e genéticas. No que se refere às áreas de concentração, diversos estudos discutem a importância da educação nutricional e da pratica regular da atividade física, os ajustes metabólicos que ocorrem nos indivíduos em programas de tratamento da obesidade, trazem elementos do âmbito das políticas públicas e questões psicossociais.
\end{abstract}

\footnotetext{
${ }^{1}$ Bacharel em Educação Física pela Universidade Estadual de Londrina. Especialista em Saúde Coletiva e Saúde da Mulher pela Faculdade UniBF. Mestrando em Educação Física pela Universidade Estadual de Londrina (UEL).

${ }^{2}$ Graduando em Medicina pela Universidade Federal do Pará (UFPA).

${ }_{3}^{3}$ Bacharela em Nutrição pela Universidade Federal do Piauí (UFPI). Pós-graduada em Nutrição Clínica, Esportiva e Prescrição de fitoterápicos e Docência do Ensino Superior pela Faculdade Adelmar Rosado.

4 Graduando em Medicina pela Universidade Federal de Pernambuco (UFPE).

${ }^{5}$ Enfermeiro. Pós graduando em Estratégia de Saúde da Família e Docência do Ensino Superior pela Faculdade Venda Nova do Imigrante (FAVENI).

${ }^{6}$ Graduando em Medicina pela Universidade de Rio Verde (UniRV).
} 


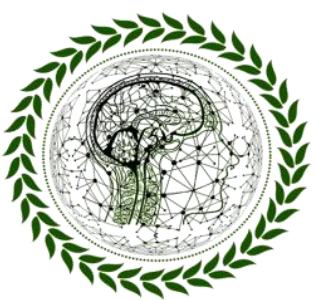

PhI Scientific Review

ISSN 2676 - 0444

Ressalta-se a necessidade dos profissionais da saúde busquem conhecimentos a respeito das prescrições, orientações e intervenções em indivíduos com obesidade, para que a intervenção seja realizada de forma eficaz e segura garantindo a saúde do indivíduo.

Palavras-chave: Obesidade. Crianças e adolescentes. Atividade física. Hábitos saudáveis.

\title{
PHYSICAL ACTIVITY, HEALTHY HABITS AND OBESITY IN CHILDREN AND ADOLESCENTS: CONSIDERATIONS, RECOMMENDATIONS AND INTERVENTIONS.
}

\begin{abstract}
Obesity is an increasingly common disease, whose prevalence is already reaching epidemic proportions. The active lifestyle and the adoption of healthy habits proved to be determinants in the current situation of childhood obesity. In this sense, obesity is considered a multifactorial disease, which should be treated in a multidisciplinary way. The objective of this work is to discuss general aspects, recommendations and interventions regarding physical activity and healthy habits in children and adolescents with obesity. This is a literature review, carried out in the Scientific Electronic Library Online (SciELO) and the Latin American and Caribbean Center for Health Science Information (Bireme) databases. The articles were selected based on the following keywords in Portuguese: childhood obesity; recommendations; care. The inclusion criteria were studies with children and adolescents who were overweight or obese that were related to the practice of physical exercise, eating habits, multiprofessional interventions. Only articles published between the years 2012 to 2020 and only in Portuguese and English were selected. In total, 12 articles composed this review. It was found that obesity is a multifactorial process that involves environmental, cultural and genetic issues. Regarding the concentration areas, several studies discuss the importance of nutrition education and regular physical activity, the metabolic adjustments that occur in individuals in obesity treatment programs, bring elements from the scope of public policies and psychosocial issues. It emphasizes the need for health professionals to seek knowledge about the prescriptions, guidelines and interventions in individuals with obesity, so that the intervention is carried out effectively and safely ensuring the health of the individual.
\end{abstract}

Keywords: Obesity. Children and teenagers. Physical activity. Healthy habits.

\section{INTRODUÇÃO}

A obesidade é uma doença cada vez mais comum, cuja prevalência já atinge proporções epidêmicas. Uma grande preocupação médica é o risco elevado de doenças associadas ao sobrepeso e à obesidade, tais como diabetes, doenças cardiovasculares (DCV) e alguns cânceres. Além disso, um quadro de obesidade pode causar incapacidade funcional, redução da qualidade de vida, redução da expectativa de vida e aumento da mortalidade. Condições crônicas, como 


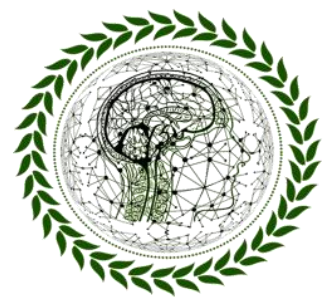

PhI Scientific Review

ISSN 2676 - 1444

doença renal, osteoartrose, câncer, DM2, apneia do sono, doença hepática gordurosa não alcoólica (DHGNA), HAS e, mais importante, DCV, estão diretamente relacionadas com incapacidade funcional e com a obesidade (ABESO, 2016).

O estilo de vida, os fatores nutricionais, as escolhas dos alimentos, quantidades e frequência com que são ingeridos mostraram-se determinantes no atual quadro da obesidade infantil. A alimentação inadequada é um fator determinante, uma vez que há a substituição de refeições balanceadas por lanches rápidos, sem valores nutricionais adequados. Afirma ainda que o nível da prática de atividade física menor do que o recomendado para uma boa saúde e a obesidade está diretamente ligado à condição obesa da criança (BERTIN et al., 2016).

A disponibilidade da tecnologia, aliada ao aumento da insegurança e a progressiva redução nos espaços livres nos centros urbanos, reduzem as oportunidades de lazer, aumentando a prática de jogar videogames e o uso dos computadores, diminuindo a prática de atividades fisicamente ativas. As crianças passam horas em frente a aparelhos de televisão e videogames, aumentando o sedentarismo e inatividade física.

Sabe-se que a prática regular de atividade física é benéfica a saúde do indivíduo, sobretudo, ao indivíduo com obesidade. Os exercícios físicos ajudam, não somente, no aprimoramento do corpo, na circulação, respiração e na musculatura, mas melhora também o humor, a qualidade do sono e a sensação de bem-estar. Um maior nível de atividade física para a criança e adolescente significa melhorar o perfil lipídico e metabólico, reduzindo, assim, a obesidade (CONDE E BORGES, 2011). Uma criança ativa tem mais probabilidade de se tornar um adulto ativo. Partindo do ponto de vista da saúde pública e medicina preventiva, a promoção de atividade física na infância e adolescência passa por estabelecer uma base sólida para a diminuição do sedentarismo na fase adulta, trazendo assim uma melhor qualidade de vida aos praticantes (CARLUCCI et al., 2013).

É bem estabelecido na literatura cientifica que o ganho de peso ocorre por diversos fatores, nesse sentido, considera-se a obesidade como uma doença multifatorial, devendo ser tratada de forma multiprofissional. Ao considera um indivíduo com um quadro de obesidade, 


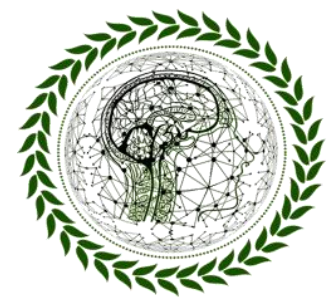

PhI Scientific Review

ISSN 2676 - 1444

deve-se compreender suas particularidades, bem como elaborar estratégias de intervenção que sejam eficazes no processo de emagrecimento visando as melhorias em saúde que a diminuição da gordura corporal pode proporcionar.

Nota-se ainda que o número de indivíduos com sobrepeso e obesidade vem crescendo significativamente no mundo todo, portanto, é necessário compreender que a obesidade se trata de um problema de saúde pública. Dada a discussão, este trabalho tem como objetivo abordar os principais tópicos desta temática, discutindo questões que envolvem recomendações e cuidados no que diz respeito as áreas da Educação Física, Nutrição, Enfermagem, Medicina e demais cursos da área da saúde compreendendo a necessidade do cuidado multiprofissional frente à crianças e adolescentes com sobrepeso e obesidade.

\section{METODOLOGIA}

O método utilizado no trabalho foi a revisão de literatura, este tipo de estudo é definido como análises da produção bibliográfica em determinada área temática, dentro de um recorte de tempo, fornecendo uma visão geral ou um relatório do sobre um tópico específico, evidenciando novas ideias, métodos, subtemas que têm recebido maior ou menor ênfase na literatura selecionada.

A presente revisão consistiu na busca de artigos científicos das bases de dados Scientific Electronic Library Online (SciELO) e Centro Latino-Americano e do Caribe de Informação em Ciências da Saúde (Bireme). Os artigos foram selecionados a partir das seguintes palavras-chave em língua portuguesa: obesidade infantil; recomendações; cuidados.

Os artigos foram selecionados sendo respeitados alguns critérios, são eles: estudos com crianças e adolescentes que apresentassem um quadro de sobrepeso ou obesidade que apresentassem relação com a prática do exercício físico, hábitos alimentares, intervenções multiprofissionais. Foram selecionados apenas artigos publicados entre os anos de 2012 a 2020 e apenas da língua portuguesa e língua inglesa. A partir das buscas realizadas nas bases de dados (SciELO e Bireme) foram encontrados um total de 12 estudos. 


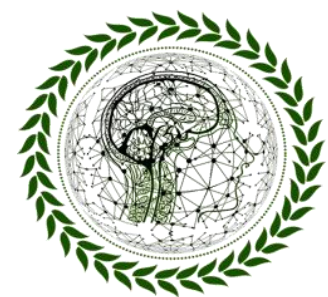

PhI Scientific Review

ISSN 2676 - 0444

O fluxograma representado abaixo caracteriza a estratégia de busca, bases de dados, critérios de inclusão e exclusão, amostra inicial, parcial e final, de acordo com a quantidade de artigos que irão compor os resultados do estudo.

Figura 1: Fluxograma do processo de identificação e seleção de artigos.

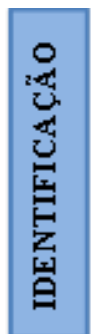

Busca em base de dados SciELO e Bireme)
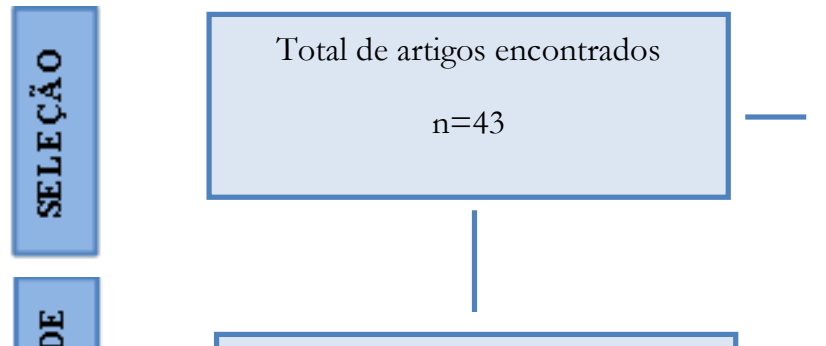

Exclusão: artigos incompletos, debates, resenhas, resumos.

$\mathrm{N}=10$

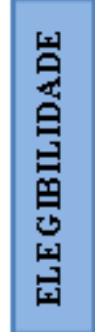

Artigos selecionados para análise na íntegra

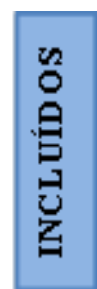

Selecionados após leitura de títulos e resumos

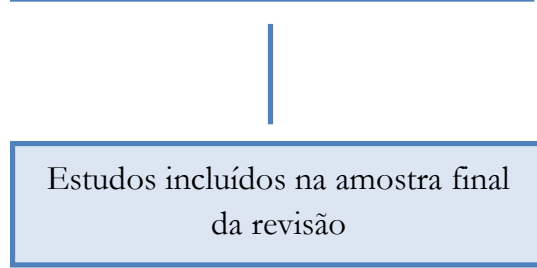

Todos os artigos selecionados estavam disponíveis online na íntegra no periódico

Fonte: Elaborado pelos autores a partir do Fluxograma PRISMA, 2021. 


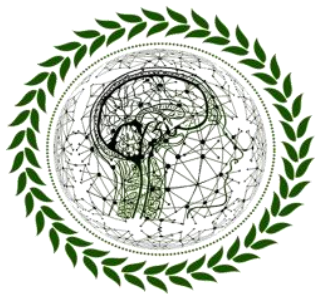

PhI Scientific Review

ISSN 2676 - 0444

3 RESULTADOS E DISCUSSÃO

Quadro 1: Artigos encontrados.

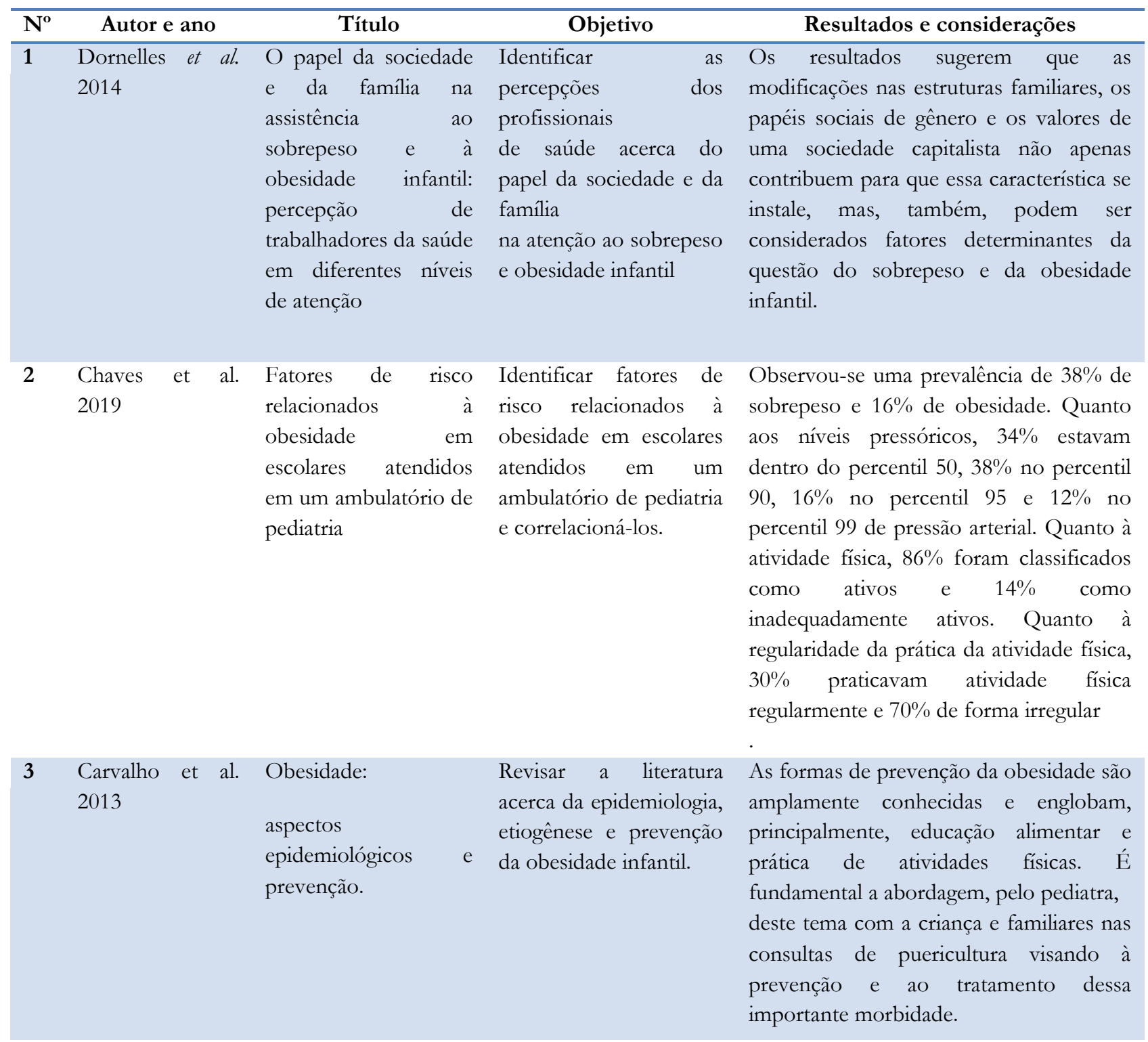

http://www.revistaphd.periodikos.com.br

V. 01, N ${ }^{o} 07$, dezembro de 2021

DOI: $10.53497 /$ phdsr1n7-003

Todos os direitos reservados (C) 


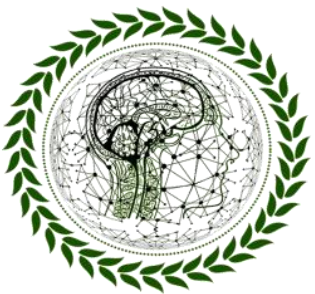

PhI Scientific Review

ISSN 2676 - 0444

4 Silva \& Bittar, Fatores Ambientais e Enfatizar os aspectos 2012

5

Silva et al. 2017

Habitos alimentares e sedentarismo

crianças

adolescentes com

obesidade na admissão do programa de obesidade do hospital universitário Bettina Ferro de Souza. ambientais na obesidade

em crianças, como importante agravo no âmbito da saúde pública.
A obesidade se apresenta não apenas como problema científico e de saúde pública, porém como grande indústria que envolve o desenvolvimento de fármacos, de alimentos modificados e estratégias governamentais estimulando a prática regular de atividade física e a orientação alimentar a fim de promover melhores hábitos.

Descrever o perfil dos hábitos alimentares e de sedentarismo em crianças e adolescentes atendidos no Centro de Referência em Obesidade Infantojuvenil do Hospital Universitário Bettina Ferro de Souza, Belém-PA.
Foram avaliados 21 pacientes, com média de idade de 7,3 anos entre as crianças e 13,8 anos entre os adolescentes. As crianças apresentaram média de peso de $45,54 \mathrm{~kg}$, IMC de $26,81 \mathrm{~kg} / \mathrm{m} 2$, e de escore-z de 4,55. Os adolescentes, peso de $71,44 \mathrm{~kg}$, IMC de $31,74 \mathrm{~kg} / \mathrm{m} 2$ e 2,58 de escore-z. Os $61,9 \%$ dos participantes tiveram baixo consumo de frutas, 95,3\% baixo consumo de hortaliças e elevado consumo de doces por $71,4 \%$. Os fisicamente inativos corresponderam a $76,2 \%$ e obteve-se alta prevalência de comportamento sedentário, com uma média de 6,42h/dia.
Linhares et al. Obesidade infantil: 2017 influência dos pais sobre a alimentação e estilo de vida dos

Filhos.
Identificar a relação da influência dos pais com a educação alimentar dos filhos, contribuindo dessa forma para o conhecimento dessa patologia.
As causas da obesidade estão diretamente relacionadas a vários fatores, podendo ser a ingestão inadequada de alimentos e falta da prática de exercícios físicos, a obesidade é também desencadeada por fatores ambientais, além de biológicos, hereditários e psicológicos.
7 Paes, Marins, Efeitos metabólicos Andreazzi, 2015 do exercício físico na obesidade infantil: uma visão atual
Revisar a literatura atual a respeito dos efeitos do exercício físico sobre diferentes variáveis metabólicas da
O exercício físico, independentemente do tipo, mostra-se capaz de promover adaptações positivas sobre a obesidade infantil, principalmente por atuar na restauração da homeostase celular e 


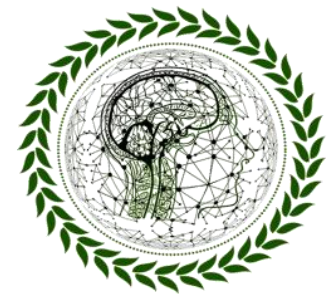

PhI Scientific Review

ISSN 2676 - 0444

obesidade infantil.

8

Peleg
2015

Pimenta, Rocha e

Marcondes, 2015

Determinar os

indicadores

antropométricos de

obesidade na predição

da gordura corporal

elevada em adolescentes de um estado brasileiro. sistema cardiovascular, na melhoria da composição corporal e também aumento da ativação metabólica.
10 Bertuol, Navarro, 2015

11 Gonçalves, Silva Variáveis psicossociais e Antunes, 2012

Consumo alimentar e prevalência de obesidade em crianças pré-escolares de uma escola infantil pública
Intervenção

de

Obesidade Infantil no

Brasil: uma Breve

Análise da Política

Nacional

de

Alimentação

e

Nutrição e Política

Nacional

Promoção da Saúde

$$
\text { de }
$$

no excesso de peso e na obesidade infantil analisar duas das principais políticas públicas intervencionistas, a Política Nacional de Alimentação e Nutrição - PNAN e a Política Nacional de Promoção da Saúde - PNPS e refletir sobre o Programa Saúde na Escola - PSE.

Avaliar o estado nutricional e o consumo alimentar de alguns produtos

industrializados, precursores da obesidade, em crianças de uma escola pública de Salvador do Sul-RS.

Avaliar as diferenças ao nível da qualidade de vida, morbilidade
Dos quatro indicadores antropométricos estudados, o IMC, a RCE e o PC tiveram as maiores áreas sob a curva ROC em relação à gordura corporal elevada relativa em ambos os sexos. Os pontos de corte para os rapazes e as moças, respectivamente, associados com gordura corporal elevada foram IMC 22,7 e 20,1 $\mathrm{kg} / \mathrm{m} 2$.

Atualmente faz-se necessário refletir sobre as Políticas Públicas e discutir suas abrangências, de modo que o maior número de interessados possa expressar seus anseios a fim de colocá-las como destaque, especialmente em momentos de participação popular.

Pré-escolares, em sua maioria são adequados diante dos dados antropométricos, mas como alimentos possuem alto consumo de açúcar e chocolate e consumo menos frequente de chips e bolo de chocolate.

Os resultados mostraram que as crianças com excesso de peso/obesidade em tratamento são as que têm uma percepção 


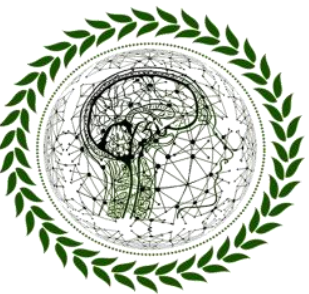

PhI Scientific Review

ISSN 2676 - (144

psicológica e imagem pessoal (autoconceito) mais negativa da sua saúde física e da saúde escolar, quando comparadas com as restantes crianças. Relativamente ao auto-conceito o grupo de crianças com excesso de peso/obesidade do grupo clínico pressiona como menos competente no domínio escolar, atlético, aparência física, aceitação social, comportamento e autoestima. As crianças do grupo clínico com excesso de peso/obesidade apresentam ainda mais sintomatologia depressiva quando comparadas com as crianças da comunidade com peso normal e com as crianças do grupo clínico.
12

Frontzek Bernardes Modena, 2017
Obesidade infantil: Compreender

para$$
\text { Compreender }
$$
melhor intervir

\section{obesidade infantil a} partir de quem vivencia o fenômeno, crianças e pais, para subsidiar intervenções mais efetivas.
Para a compreensão da obesidade infantil e para se pensar em intervenções mais eficazes deve-se considerar o sentido atribuído à obesidade, as relações familiares, as condições socioeconômicas e todos os elementos que circundam a obesidade infantil.

Fonte: Elaborado pelos autores (2021).

Os artigos que foram selecionados trazem diferentes abordagens, tendo em vista que a obesidade se trata de um processo multifatorial, complexo, que envolve questões ambientais, culturais e genéticas. No que se refere às áreas de concentração, diversos estudos discutem a importância da educação nutricional e da pratica regular da atividade física, os ajustes metabólicos que ocorrem nos indivíduos em programas de tratamento da obesidade, trazem elementos do âmbito das políticas públicas e questões psicossociais.

As estratégias de tratamento da obesidade e do sobrepeso infantil são pouco documentadas, comparando-se com os trabalhos existentes sobre adultos. Apesar de não haver tratamento considerado padrão, pela inconclusividade derivada de problemas metodológicos frequentemente encontrados nos trabalhos disponíveis, as recomendações atuais para o manejo 


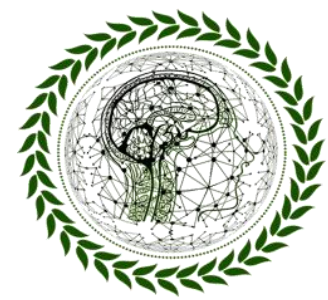

PhI Scientific Review

ISSN 2676 - (144

clínico do excesso de peso em crianças e adolescentes baseiam-se no controle de ganho ponderal e das comorbidades eventualmente encontradas (Escobar, 2009); (Machado et al., 2012).

O tratamento convencional fundamenta-se na redução da ingestão calórica, aumento do gasto energético, modificação comportamental e envolvimento familiar no processo de mudança. O tratamento se dá em longo prazo e sugerem-se visitas frequentes.

O tratamento dietético deve focar-se na adequação da ingestão calórica e no suprimento das necessidades nutricionais para a idade. A proporção calórica dos macronutrientes deve seguir a recomendação das diretrizes nacionais e internacionais de alimentação saudável. Do total de calorias da dieta, $15 \%$ devem provir de proteínas, $50 \%$ a $55 \%$, dos carboidratos e $30 \%$, das gorduras.

No que se refere a utilização de outras estratégias, tais como dietas de maior restrição calórica, tratamento medicamentoso ou cirurgia bariátrica, no tratamento da obesidade em adolescentes, quando esta se apresentar em graus mais graves e se associar a comorbidades importantes. O tratamento clínico é baseado em modificações do estilo de vida e pode incluir o uso de medicamentos (Soares et al., 2018).

Uma intervenção terciária de tratamento deve ser usada apenas de forma limitada na população pediátrica, para adolescentes severamente obesos incapazes de diminuir a adiposidade e reduzir seus riscos de morbidade, suficientemente maduros para entender os possíveis riscos associados. Essa intervenção pode considerar um protocolo de substituição de refeições com dieta de muito baixas calorias, medicação antiobesidade e, em último caso, cirurgia bariátrica. Os riscos e benefícios dessas condutas devem ser cuidadosamente avaliados, caso a caso, até que se obtenha maior nível de evidência. Só se deve considerar tais alternativas no tratamento de crianças e adolescentes que não responderam ao tratamento convencional e apresentam graves comorbidades associadas ao excesso de peso. 


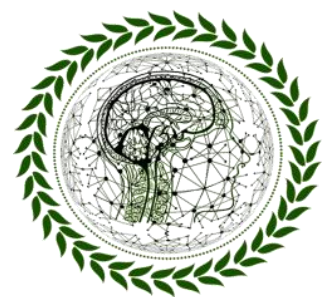

PhI Scientific Review

ISSN 2676 - (144

\section{CONSIDERAÇÕES FINAIS}

A obesidade como fenômeno multifatorial, algumas considerações devem ser trazidas, como compreender os aspectos gerais da doença para que novas discussões sejam realizadas com o propósito de salientar a população os diversos malefícios que a obesidade pode acarretar a saúde do indivíduo. Nesse sentido, é necessário que existe uma maior mobilização por parte dos profissionais da saúde para que as recomendações da prática da atividade física, a adoção de hábitos saudáveis e o tratamento multiprofissional ocorra de forma eficaz.

O trabalho propôs apresentar considerações, recomendações e estratégias de intervenção para o combate a obesidade. Nota-se grande variedade de estudos que abordam a importância bem como os benefícios do exercício físico e da alimentação saudável para essa população. $\mathrm{O}$ cuidado multiprofissional parece ser uma importante estratégia para a diminuição dos prejuízos causados pelas alterações fisiológicas e psicossociais observadas em indivíduos com sobrepeso e obesidade.

Por fim, considera-se a temática de grande relevância, uma vez que busca através da literatura científica respostas para questões que ainda são consideradas "tabus" na sociedade. Ressalta-se a necessidade dos profissionais da saúde busquem conhecimentos a respeito das prescrições, orientações e intervenções em indivíduos com obesidade, para que a intervenção seja realizada de forma eficaz e segura garantindo a saúde do indivíduo.

\section{REFERÊNCIAS}

ABESO. Diretrizes brasileiras de obesidade 2016. ABESO - Associação Brasileira para o Estudo da Obesidade e da Síndrome Metabólica. - 4.ed. - São Paulo, SP. 2016.

BERTIN, R.L. et al. Percepções do cotidiano alimentar de crianças e adolescents com diabetes mellitus tipo 1. Revista Contexto \& Saúde, Ijuí v. 16 n. 2016. 


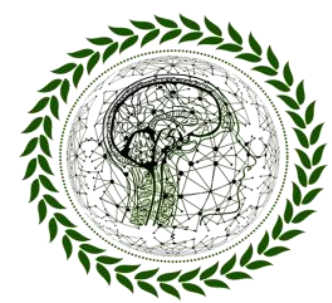

PhI Scientific Review

ISSN 2676 - 0444

CARLUCCI, E.M.S. et al. Obesidade e sedentarismo: fatores de risco para doença cardiovascular. Com. Ciências Saúde. 2013; 24(4): 375-384.

CONDE, W.L. e BORGES, C. O risco de incidência e persistência da obesidade entre adultos brasileiros segundo seu estado nutricional ao final da adolescência. Rev Bras. Epidemiol. 2011; 14(1) Supl.: 71-9.

ESCOBAR F.A. Relação entre Obesidade e Diabete Mellitus Tipo II em Adultos. Cadernos UNIFOA edição nº 11, 2009.

MACHADO, S.P. et al. Correlação entre o índice de massa corporal e indicadores antropométricos de obesidade abdominal em portadores de diabetes mellitus tipo 2. Rev. Bras. Promoç. Saúde, Fortaleza, 25(4): 512-520., 2012.

SOARES G.C. et al. Os fatores que influenciam na obesidade infantil: uma revisão da literatura. Conselho Federal de Enfermagem. Disponível em: http://apps.cofen.gov.br/cbcenf/sistemainscricoes/arquivosTrabalhos/I40163.E10.T7059.D 6AP.pdf. 